Global Journal of Pure and Applied Mathematics.

ISSN 0973-1768 Volume 13, Number 6 (2017), pp. 1853-1866

(C) Research India Publications

https://dx.doi.org/10.37622/GJPAM/13.6.2017.1853-1866

\title{
On the analysis of Hilbert module-valued functions on LCA groups
}

\author{
Mawoussi Todjro ${ }^{1}$ \\ Department of Mathematics, \\ University of Lomé, P.O.Box 1515, Lomé, Togo. \\ Yaogan Mensah \\ Department of Mathematics, \\ University of Lomé, P.O.Box 1515, Lomé, Togo \\ and International Chair in Mathematical Physics and \\ Applications-Unesco Chair, \\ University of Abomey-Calavi, Cotonou, Benin. \\ Kokou Tcharie \\ Department of Mathematics, \\ University of Lomé, P.O.Box 1515, Lomé, Togo.
}

\begin{abstract}
Let $\mathcal{M}$ be a Hilbert module over a $C^{*}$-algebra $\mathcal{A}$. This paper adresses some facts about functions in the space $L_{2}(G, \mathcal{M})$ of Bochner-square integrable Hilbert module-valued functions on the locally compact abelian group $G$. We construct an action of $\mathcal{A}$ over $L_{2}(G, \mathcal{M})$ and endow the latter with a pre-Hilbert module structure. We also prove some results of Parseval type.
\end{abstract}

AMS subject classification: Primary 43A25, 43A15; Secondary 28B05, 46C50, 46L08.

Keywords: Hilbert $C^{*}$-module, locally compact abelian group, Fourier transform, square-integrable function.

\footnotetext{
${ }^{1}$ Corresponding author.
} 


\section{Introduction}

First introduced by I. Kaplansky in 1953 [5], Hilbert $C^{*}$-modules are natural generalization of Hilbert spaces. They are linear spaces endowed with an inner product which values occur in a $C^{*}$-algebra. The subject has been deeply explored and extended by W. L. Paschke [10], M. Rieffel [11], Kasparov [6]. Nowadays, Hilbert $C^{*}$-module is intensevily present in research [1], [4], [14], [15] and applications particularly in harmonic analysis [9]. The main purpose of this paper is to contribute to consolidate the link between Hilbert $C^{*}$-modules and Harmonic analysis. We are mainly interested in some properties of Hilbert $C^{*}$-module valued functions on locally compact abelian groups in connection with the Fourier transform.

The paper is organized as follow. The next section recalls some definitions and properties of notions like Hilbert module and Pontryagin duality. The section 3 is devoted to our main results.

\section{Preliminaries}

\subsection{Hilbert modules}

If $\mathcal{M}$ is a module over $\mathcal{A}$, the action of an element $a \in \mathcal{A}$ on an element $x$ of $\mathcal{M}$ is denoted by $x \cdot a$.

Definition 2.1. A pre-Hilbert module over a $C^{*}$-algebra $\mathcal{A}$ is a (right) $\mathcal{A}$-module $\mathcal{M}$ equipped with a sesquilinear map $\langle\cdot, \cdot\rangle: \mathcal{M} \times \mathcal{M} \rightarrow \mathcal{A}$ with the following properties:

1. $\forall x, y, z \in \mathcal{M}, \forall \alpha, \beta \in \mathbb{C},\langle x, \alpha y+\beta z\rangle=\alpha\langle x, y\rangle+\beta\langle x, z\rangle$,

2. $\forall x, y \in \mathcal{M}, \forall a \in \mathcal{A},\langle x, y \cdot a\rangle=\langle x, y\rangle a$,

3. $\forall x, y \in \mathcal{M},\langle y, x\rangle=\langle x, y\rangle^{*}$,

4. $\forall x \in \mathcal{M},\langle x, x\rangle \geq 0$ and if $\langle x, x\rangle=0$ then $x=0$.

The map $\langle\cdot, \cdot\rangle$ is called an $\mathcal{A}$-valued inner product.

Let $\mathcal{M}$ be a pre-Hilbert module over $\mathcal{A}$ endowed with a norm $\|\cdot\|_{\mathcal{A}}$. For $x \in \mathcal{M}$, set $\|x\|_{\mathcal{M}}=\|\langle x, x\rangle\|_{\mathcal{A}}^{\frac{1}{2}}$.

Proposition 2.2. The map $x \mapsto\|x\|_{\mathcal{M}}$ is a norm on $\mathcal{M}$ and satisfies the following properties:

1. $\|x \cdot a\|_{\mathcal{M}} \leqslant\|x\|_{\mathcal{M}}\|a\|_{\mathcal{A}}$ for all $x \in \mathcal{M}, a \in \mathcal{A}$;

2. $\|\langle x, y\rangle\|_{\mathcal{A}} \leqslant\|x\|_{\mathcal{M}}\|y\|_{\mathcal{M}}$ for all $x, y \in \mathcal{M}$. 
If $\mathcal{M}$ is a Banach space under the norm $\|\cdot\|_{\mathcal{M}}$, then $\mathcal{M}$ is called a Hilbert $\mathcal{A}$-mdoule (or Hilbert $C^{*}$-module over $\mathcal{A}$ ).

Here are some simple examples of Hilbert modules.

\section{Example 2.3.}

1. Every complex Hilbert space is a Hilbert $C^{*}$-module over $\mathbb{C}$.

2. Every $C^{*}$-algebra $\mathcal{A}$ is a Hilbert module over $\mathcal{A}$. The $\mathcal{A}$-valued inner product is given by $\langle a, b\rangle=a^{*} b, \forall a, b \in \mathcal{A}$. A concrete example is $\mathcal{B}(H)$ the set of bounded operators on a Hilbert space $H$.

\subsection{LCA group, Pontryagin duality and Fourier transform}

Let $G$ be a locally compact abelian group and $\lambda$ its Haar measure. We denote by $\widehat{G}$ the dual group of $G$, which is group of all continuous group homomorphisms $\chi: G \rightarrow \mathbb{T}$ where $\mathbb{T}$ is the circle group, [2]. These group homomorphisms are called characters of $G$. The dual group $\widehat{G}$ is an abelian group under the point-wise multiplication

$$
(\chi \cdot \eta)(g)=\chi(g) \cdot \eta(g), \text { for all } g \in G .
$$

The inverse element to a given character $\chi \in \widehat{G}$ is defined by

$$
\chi^{-1}(g)=(\chi(g))^{-1}=\overline{\chi(g)}, \text { for all } g \in G .
$$

It is well-known that $\widehat{G}$ carries a natural topology called the compact-open topology [7], [13] under which $\widehat{G}$ is a locally compact group. Thereby, it admits a Haar measure which we denote $\Lambda$.

We denote $\widehat{\widehat{G}}$ the dual group of $\widehat{G}$. The Pontryagin duality states that there exists a canonical and topological isomorphism of groups between $G$ and $\widehat{\widehat{G}}$. This isomorphism is given by

$$
\delta: G \rightarrow \widehat{\widehat{G}}, \quad g \mapsto \delta_{g}
$$

where

$$
\delta_{g}: \widehat{G} \rightarrow \mathbb{C}, \quad \chi \mapsto \delta_{g}(\chi)=\chi(g) .
$$

If $G$ is a compact abelian group, [3] we have for all $\chi, \eta \in \widehat{G}$,

$$
\langle\chi, \eta\rangle_{\widehat{G}}=\int_{G} \chi(g) \overline{\eta(g)} d g=\left\{\begin{array}{l}
1 \text { if } \chi=\eta \\
0 \text { otherwise }
\end{array}\right.
$$

If $\widehat{G}$ is a compact abelian group, we have for all $u, v \in G$,

$$
\left\langle\delta_{u}, \delta_{v}\right\rangle_{\widehat{G}}=\int_{\widehat{G}} \delta_{u}(\chi) \overline{\delta_{v}(\chi)} d \Lambda(\chi)=\delta_{u, v}
$$

where $\delta_{u, v}$ denotes Kronecker delta. 
We denote respectively $L_{1}(G, \mathcal{M}), L_{2}(G, \mathcal{M})$ and $L_{2}(\widehat{G}, \mathcal{M})$ the space of the Bochner-integrable $\mathcal{M}$-valued functions on $G$, the space of Bochner-square integrable $\mathcal{M}$-valued functions on $G$ and the space of Bochner-square integrable $\mathcal{M}$-valued functions on $\widehat{G}$.

Let $f \in L_{1}(G, \mathcal{M})$, the Fourier transform of $f$ is defined by

$$
\widehat{f}(\chi)=\int_{G} f(u) \overline{\chi(u)} d \lambda(u) \text { for all } \chi \in \widehat{G} .
$$

\section{Main results}

In this section $G$ is a locally compact abelian group unless otherwise stated. For $f, g \in$ $L_{2}(G, \mathcal{M})$. Set

$$
\langle f, g\rangle_{L_{2}(G, \mathcal{M})}=\int_{G}\langle f(t), g(t)\rangle d \lambda(t) .
$$

Likewise, for $\psi, \phi \in L_{2}(\widehat{G}, \mathcal{M})$, set

$$
\left.\langle\phi, \psi\rangle_{L_{2}(\widehat{G}, \mathcal{M})}\right)=\int_{\widehat{G}}\langle\phi(\chi), \psi(\chi)\rangle d \Lambda(\chi) .
$$

On the other hand, for all $f \in L_{1}(G, \mathcal{M})$ and $a \in \mathcal{A}$, set

$$
f \cdot a: G \rightarrow \mathcal{M}, \quad t \mapsto f(t) a .
$$

And for all $\phi \in L_{1}(\widehat{G}, \mathcal{M})$ and $a \in \mathcal{A}$, set

$$
\phi \cdot a: \widehat{G} \rightarrow \mathcal{M}, \quad \chi \mapsto \phi(\chi) a .
$$

Proposition 3.1. The map $\mathcal{A} \times L_{2}(G, \mathcal{M}) \rightarrow L_{2}(G, \mathcal{M}),(a, f) \mapsto f \cdot a$ is an action of $\mathcal{A}$ on $L_{2}(G, \mathcal{M})$.

Proof. Let $f \in L_{2}(G, \mathcal{M})$ and $a \in \mathcal{A}$, we have $f \cdot a \in L_{2}(G, \mathcal{M})$ since

$$
\begin{aligned}
\int_{G}\|(f \cdot a)(t)\|_{\mathcal{M}}^{2} d \lambda(t) & =\int_{G}\|f(t) a\|_{\mathcal{M}}^{2} d \lambda(t) \\
& =\int_{G}\|\langle f(t) a, f(t) a\rangle\|_{\mathcal{A}} d \lambda(t) \\
& =\int_{G}\left\|a^{*}\langle f(t), f(t)\rangle_{a}\right\|_{\mathcal{A}} d \lambda(t) \\
& \leq \int_{G}\left\|a^{*}\right\| \mathcal{A}\|f(t)\|_{\mathcal{M}}^{2}\|a\|_{\mathcal{A}} d \lambda(t) \\
& =\int_{G}\|f(t)\|_{\mathcal{M}}^{2}\|a\|_{\mathcal{A}}^{2} d \lambda(t) \\
& =\left(\int_{G}\|f(t)\|_{\mathcal{M}}^{2} d \lambda(t)\right)\|a\|_{\mathcal{A}}^{2}<+\infty
\end{aligned}
$$


Furthermore, for any $t \in G$ and $a, b \in \mathcal{A}$ we have

$$
((f \cdot a) \cdot b)(t)=(f \cdot a)(t) b=(f(t) a) b=f(t)(a b)=(f \cdot(a b))(t) .
$$

Hence $(f \cdot a) \cdot b=f \cdot(a b)$.

Proposition 3.2. The map $L_{2}(G, \mathcal{M}) \times L_{2}(G, \mathcal{M}) \rightarrow \mathcal{A},(f, g) \mapsto\langle f, g\rangle_{L_{2}(G, \mathcal{M})}$ is an $\mathcal{A}$-valued inner product.

Proof. Let $f, g \in L_{2}(G, \mathcal{M})$ and $a \in \mathcal{A}$.

1. Then $\|f(\cdot)\|_{\mathcal{M}}$ and $\|g(\cdot)\|_{\mathcal{M}}$ belong to $L_{2}(G, \mathbb{C})$, so $\|f(\cdot)\|_{\mathcal{M}}\|g(\cdot)\|_{\mathcal{M}} \in L_{1}(G, \mathbb{C})$. We have

$\int_{G}\|\langle f(t), g(t)\rangle\|_{\mathcal{A}} d \lambda(t) \leq \int_{G}\|f(t)\|_{\mathcal{M}}\|g(t)\|_{\mathcal{M}} d \lambda(t)<+\infty$. Hence $\langle\cdot, \cdot\rangle_{L_{2}(G, \mathcal{M})}$ is well defined.

2. Let $\alpha, \beta \in \mathbb{C}$. We have

$$
\langle f, \alpha g+\beta h\rangle_{L_{2}(G, \mathcal{M})}=\int_{G}\langle f(t), \alpha g(t)+\beta h(t)\rangle d \lambda(t)=\alpha\langle f, g\rangle_{L}+\beta\langle f, h\rangle_{L_{2}(G, \mathcal{M})} .
$$

3. We have

$$
\langle f, g \cdot a\rangle_{L_{2}(G, \mathcal{M})}=\int_{G}\langle f(t), g(t) a\rangle d \lambda(t)=\int_{G}\langle f(t), g(t)\rangle a d \lambda(t)=\langle f, g\rangle_{L_{2}(G, \mathcal{M})} a .
$$

4. We have

$$
\begin{aligned}
\langle f, g\rangle_{L_{2}(G, \mathcal{M})} & =\int_{G}\langle f(t), g(t)\rangle d \lambda(t)=\int_{G}\langle g(t), f(t)\rangle^{*} d \lambda(t) \\
& =\left(\int_{G}\langle g(t), f(t)\rangle d \lambda(t)\right)^{*}=\langle g, f\rangle_{L_{2}(G, \mathcal{M})}^{*} .
\end{aligned}
$$

5. We have $\langle f, f\rangle_{L_{2}(G, \mathcal{M})}=\int_{G}\langle f(t), f(t)\rangle d \lambda(t) \geq 0$ since $\forall t \in G,\langle f(t), f(t)\rangle \geq$ 0 . Furthermore; let $f \in L_{2}(G, \mathcal{M})$ such that $\int_{G}\langle f(t), f(t)\rangle d \lambda(t)=0$, then $t \mapsto\langle f(t), f(t)\rangle$ is null $\lambda$-a.e., Therefore $f=0 \lambda$-a.e.. So $\langle f, f\rangle_{L_{2}(G, \mathcal{M})}=0$ gives $f=0$ for $f \in L_{2}(G, \mathcal{M})$.

Corollary 3.3. The space $L_{2}(G, \mathcal{M})$ equipped with the above $\mathcal{A}$-valued inner product $\langle\cdot, \cdot\rangle_{L_{2}(G, \mathcal{M})}$ is a pre-Hilbert module over $\mathcal{A}$.

Proof. We only need to show that $L_{2}(G, \mathcal{M})$ is a complex vector space. 
Let $f, g \in L_{2}(G, \mathcal{M})$, we have

$$
\begin{aligned}
\int_{G}\|(f+g)(t)\|_{\mathcal{M}}^{2} d \lambda(t)= & \int_{G}\|\langle f(t)+g(t), f(t)+g(t)\rangle\|_{\mathcal{A}} d \lambda(t) \\
\leqslant & \int_{G}\|\langle f(t), f(t)\rangle\|_{\mathcal{A}} d \lambda(t)+\int_{G}\|\langle f(t), g(t)\rangle\|_{\mathcal{A}} d \lambda(t) \\
& +\int_{G}\|\langle g(t), f(t)\rangle\|_{\mathcal{A}} d \lambda(t)+\int_{G}\|\langle g(t), g(t)\rangle\|_{\mathcal{A}} d \lambda(t) .
\end{aligned}
$$

The integral

$$
\int_{G}\|\langle f(t), f(t)\rangle\|_{\mathcal{A}} d \lambda(t)=\int_{G}\|f(t)\|_{\mathcal{M}}^{2} d \lambda(t)
$$

is finite. And it is the same to

$$
\int_{G}\|\langle g(t), g(t)\rangle\|_{\mathcal{A}} d \lambda(t)
$$

By Hölder's inequality,

$$
\begin{aligned}
\int_{G}\|\langle f(t), g(t)\rangle\|_{\mathcal{A}} d \lambda(t) & \leqslant \int_{G}\|f(t)\|\left\|_{\mathcal{M}}\right\| g(t) \|_{\mathcal{M}} d \lambda(t) \\
& \leqslant\left(\int_{G}\|f(t)\|_{\mathcal{M}}^{2} d \lambda(t)\right)^{\frac{1}{2}}\left(\int_{G}\|g(t)\|_{\mathcal{M}}^{2} d \lambda(t)\right)^{\frac{1}{2}}<+\infty .
\end{aligned}
$$

Similary

$$
\int_{G}\|\langle g(t), f(t)\rangle\|_{\mathcal{A}} d \lambda(t)<+\infty
$$

Hence

$$
\int_{G}\|(f+g)(t)\|_{\mathcal{M}}^{2} d \lambda(t)<+\infty \text { and } f+g \in L_{2}(G, \mathcal{M}) .
$$

Moreover for all $f \in L_{2}(G, \mathcal{M})$ and $\alpha \in \mathbb{C}, \alpha f \in L_{2}(G, \mathcal{M})$.

Proposition 3.4. The map $\mathcal{A} \times L_{2}(\widehat{G}, \mathcal{M}) \rightarrow L_{2}(\widehat{G}, \mathcal{M}),(a, \phi) \mapsto \phi \cdot a$ is an action of $\mathcal{A}$ on $L_{2}(\widehat{G}, \mathcal{M})$.

Proof. Let $\phi \in L_{2}(\widehat{G}, \mathcal{M})$ and $a \in \mathcal{A}$. We have

$$
\begin{aligned}
\int_{\widehat{G}}\|(\phi \cdot a)(\chi)\|_{\mathcal{M}}^{2} d \Lambda(\chi) & =\int_{\widehat{G}}\|\phi(\chi) a\|_{\mathcal{M}}^{2} d \Lambda(\chi) \\
& \leqslant \int_{\widehat{G}}\|\phi(\chi)\|_{\mathcal{M}}^{2}\|a\|_{\mathcal{A}}^{2} d \Lambda(\chi) \\
& =\left(\int_{\widehat{G}}\|\phi(\chi)\|_{\mathcal{M}}^{2} d \Lambda(\chi)\right)\|a\|_{\mathcal{A}}^{2}<+\infty
\end{aligned}
$$


Moreover, if $\phi \in L_{2}(\widehat{G}, \mathcal{M})$ and $a, b \in \mathcal{A}$, then $((\phi \cdot a) \cdot b)(\chi)=((\phi \cdot a)(\chi)) b=$ $((\phi(\chi) a) b=\phi(\chi)(a b)=(\phi \cdot(a b))(\chi)$ for all $\chi \in \widehat{G}$.

Proposition 3.5. The map $L_{2}(\widehat{G}, \mathcal{M}) \times L_{2}(\widehat{G}, \mathcal{M}) \rightarrow \mathcal{A},(\phi, \psi) \mapsto\langle\phi, \psi\rangle_{L_{2}(\widehat{G}, \mathcal{M})}$ is an $\mathcal{A}$-valued inner product.

Proof. It is similar to the proof of the Proposition 3.2.

Corollary 3.6. The space $L_{2}(\widehat{G}, \mathcal{M})$ equipped with the above $\mathcal{A}$-valued inner product $\langle\cdot, \cdot\rangle_{L_{2}(\widehat{G}, \mathcal{M})}$ is a pre-Hilbert module over $\mathcal{A}$.

Proof. The proof is the same as that of the corollary 3.3.

Proposition 3.7. Let $f \in L_{1}(G, \mathcal{M})$, then $\sup _{\chi \in \widehat{G}}\|\widehat{f}(\chi)\|_{\mathcal{M}} \leqslant\|f\|_{1}$.

Proof. Let $f \in L_{1}(G, \mathcal{M})$. We have

$$
\begin{aligned}
\|\widehat{f}(\chi)\|_{\mathcal{M}} & =\left\|\int_{G} \overline{\chi(t)} f(t) d \lambda(t)\right\|_{\mathcal{M}} \\
& \leqslant \int_{G}\|\overline{\chi(t)} f(t)\|_{\mathcal{M}} d \lambda(t) \\
& =\int_{G} \mid \overline{\chi(t)}\|\| f(t) \|_{\mathcal{M}} d \lambda(t) \\
& =\int_{G}\|f(t)\|_{\mathcal{M}} d \lambda(t) \\
& =\|f\|_{1} .
\end{aligned}
$$

Hence

$$
\sup _{\chi \in \widehat{G}}\|\widehat{f}(\chi)\|_{\mathcal{M}} \leqslant\|f\|_{1}
$$

We designate by $\mathcal{C}_{0}(\widehat{G}, \mathcal{M})$ the space of continuous $\mathcal{M}$-valued functions on $\widehat{G}$ which vanish at infinity and by $\mathcal{C}_{c}(G, \mathcal{M})$ and $\mathcal{C}_{c}(\widehat{G}, \mathcal{M})$ the spaces of continuous $\mathcal{M}$-valued functions with compact support in $G$ and $\widehat{G}$ respectively.

Let $K$ be compact set of $G$ and $r>0$, put

$$
U_{r}=\{z \in \mathbb{C} ;|1-z|<r\} \text { and } N_{K}(r)=\left\{\chi \in \widehat{G} ; \chi(t) \in U_{r} \text { for all } t \in K\right\} .
$$

The set $\left\{N_{K}(r) ; K\right.$ compact of $G$ and $\left.r>0\right\}$ is a family of open sets of $\widehat{G}$ [12] and is better taken as a basis of neighbourhoods of the unit character.

Proposition 3.8. Let $f \in L_{1}(G, \mathcal{M})$ then $\widehat{f} \in \mathcal{C}_{0}(\widehat{G}, \mathcal{M})$. 
Proof. Let $f \in \mathcal{C}_{c}(G, \mathcal{M})$. Let us show that $\widehat{f}$ is uniformly continuous. Let $\epsilon>0$ and $\chi, \eta \in \widehat{G}$. Since the Haar measure $\lambda$ is regular, there exists a compact set $K \subset G$ such that $|\lambda|(G-K)<\frac{\epsilon}{4 \sup _{G}\|f(t)\|_{\mathcal{M}}}$.

We have

$$
\begin{aligned}
\|\widehat{f}(\chi)-\widehat{f}(\eta)\|_{\mathcal{M}}= & \left\|\int_{G}(\overline{\chi(t)}-\overline{\eta(t)}) f(t) d \lambda(t)\right\|_{\mathcal{M}} \\
\leqslant & \left.\int_{G} \| \overline{\chi(t)}-\overline{\eta(t)}\right) f(t) \|_{\mathcal{M}} d \lambda(t) \\
= & \int_{G}\|f(t)\|_{\mathcal{M}}|\chi(t)-\eta(t)| d \lambda(t) \\
= & \int_{K}\|f(t)\|_{\mathcal{M}}|\chi(t)-\eta(t)| d \lambda(t) \\
& +\int_{G-K}\|f(t)\|_{\mathcal{M}}|\chi(t)-\eta(t)| d \lambda(t) \\
= & \int_{K}\|f(t)\|_{\mathcal{M}}\left|1-\chi^{-1}(t) \eta(t)\right| d \lambda(t) \\
& +\int_{G-K}\|f(t)\|_{\mathcal{M}}|\chi(t)-\eta(t)| d \lambda(t) .
\end{aligned}
$$

If $\chi^{-1} \eta \in N_{K}\left(\frac{\epsilon}{2\|f\|_{1}}\right)$ then $\left|1-\chi^{-1}(t) \eta(t)\right|<\frac{\epsilon}{2\|f\|_{1}}$. First, one has

$$
\int_{K}\|f(t)\|_{\mathcal{M}}\left|1-\chi^{-1}(t) \eta(t)\right| d \lambda(t) \leqslant \frac{\epsilon}{2\|f\|_{1}} \int_{K}\|f(t)\|_{\mathcal{M}} d \lambda(t) \leqslant \frac{\epsilon}{2} .
$$

And thereafter one has

$$
\begin{aligned}
\int_{G-K}\|f(t)\|_{\mathcal{M}}|\chi(t)-\eta(t)| d \lambda(t) & \leqslant \int_{G-K}\|f(t)\|_{\mathcal{M}}(|\chi(t)|+|\eta(t)|) d \lambda \\
& =2 \int_{G-K}\|f(t)\|_{\mathcal{M}} d \lambda(t) \\
& \leqslant 2 \int_{G-K}\|f(t)\|_{\mathcal{M} d|\lambda|(t)} \\
& \leqslant 2 \sup _{G}\|f(t)\|_{\mathcal{M}|\lambda|(G-K)} \\
& \leqslant \frac{\epsilon}{2}
\end{aligned}
$$

Hence $\forall \chi, \eta \in \widehat{G}$, if $\chi^{-1} \eta \in N_{K}\left(\frac{\epsilon}{2\|f\|_{1}}\right)$ then

$$
\|\widehat{f}(\chi)-\widehat{f}(\eta)\|_{\mathcal{M}}<\frac{\epsilon}{2}+\frac{\epsilon}{2}=\epsilon .
$$


We deduce that $\widehat{f}$ is uniformly continuous.

Let $f \in L_{1}(G, \mathcal{M})$, since $\mathcal{C}_{c}(G, \mathcal{M})$ is dense in $L_{1}(G, \mathcal{M})$, for all $\epsilon>0$, there exists $g \in \mathcal{C}_{c}(G, \mathcal{M})$ such that $\|f-g\|_{1}<\frac{\epsilon}{3}$. Let $\chi, \eta \in \widehat{G}$ be such that $\chi^{-1} \eta \in N_{K}\left(\frac{\epsilon}{2\|f\|_{1}}\right)$. We have

$$
\|\widehat{f}(\chi)-\widehat{f}(\eta)\|_{\mathcal{M}} \leqslant\|\widehat{f}(\chi)-\widehat{g}(\chi)\|_{\mathcal{M}}+\|\widehat{g}(\chi)-\widehat{g}(\eta)\|_{\mathcal{M}}+\|\widehat{f}(\eta)-\widehat{g}(\eta)\|_{\mathcal{M}}
$$

From the proposition 3.7, we have $\|\widehat{f}(\theta)-\widehat{g}(\theta)\|_{\mathcal{M}} \leqslant\|f-g\|_{1} \leqslant \frac{\epsilon}{3}$ for all $\theta \in \widehat{G}$. On the other hand we have $\|\widehat{g}(\chi)-\widehat{g}(\eta)\|_{\mathcal{M}} \leqslant \frac{\epsilon}{3}$. Therefore $\|\widehat{f}(\chi)-\widehat{f}(\eta)\|_{\mathcal{M}} \leqslant \epsilon$. Hence $\widehat{f}$ is uniformly continuous on $\widehat{G}$.

Finally, since $\widehat{f}$ is continuous map, there exists a sequence $\phi_{n}$ in $\mathcal{C}_{c}(\widehat{G}, \mathcal{M})$ which converges to $\widehat{f}$. For all $\chi \in \widehat{G}$, one has $\left\|\phi_{n}(\chi)-\hat{f}(\chi)\right\|_{\mathcal{M}} \leqslant\left\|\phi_{n}-\widehat{f}\right\|_{1}$. Hence $\phi_{n}$ converges uniformly to $\hat{f}$. Therefore $\widehat{f} \in \mathcal{C}_{0}(\widehat{G}, \mathcal{M})$.

Proposition 3.9. Let $G$ be a compact abelian group, then for $f, g \in L_{2}(G, \mathcal{M})$, one has

$$
\langle\widehat{f}, \widehat{g}\rangle_{L_{2}(\widehat{G}, \mathcal{M})}=\langle f, g\rangle_{L_{2}(G, \mathcal{M})} .
$$

Proof. We have:

$$
\begin{aligned}
\langle\widehat{f}, \widehat{g}\rangle_{L_{2}(\widehat{G}, \mathcal{M})} & =\int_{\widehat{G}}\langle\widehat{f}(\chi), \widehat{g}(\chi)\rangle d \Lambda(\chi) \\
& =\int_{\widehat{G}}\left\langle\int_{G} f(u) \overline{\chi(u)} d \lambda(u), \int_{G} g(v) \overline{\chi(v)} d \lambda(v)\right\rangle d \Lambda(\chi) \\
& =\int_{\widehat{G}}\left(\int_{G} \int_{G}\langle f(u) \overline{\chi(u)}, g(v) \overline{\chi(v)}\rangle d \lambda(u) d \lambda(v)\right) d \Lambda(\chi) \\
& =\int_{G} \int_{G}\left(\langle f(u), g(v)\rangle \int_{\widehat{G}} \chi(u) \overline{\chi(v)} d \Lambda(\chi)\right) d \lambda(u) d \lambda(v) \\
& =\int_{G} \int_{G}\left(\langle f(u), g(v)\rangle \int_{\widehat{G}} \delta_{u}(\chi) \overline{\delta_{v}(\chi)} d \Lambda(\chi)\right) d \lambda(u) d \lambda(v) \\
& =\int_{G} \int_{G}\langle f(u), g(v)\rangle\left\langle\delta_{u}, \delta_{v}\right\rangle \widehat{\widehat{G}} d \lambda(u) d \lambda(v) \\
& =\int_{G} \int_{G}\langle f(u), g(v)\rangle \delta_{u, v} d \lambda(u) d \lambda(v) \\
& =\int_{G}\langle f(v), g(v)\rangle d \lambda(v) \\
& =\langle f, g\rangle_{L_{2}(G, \mathcal{M}) .}
\end{aligned}
$$

We have the following norms

$$
\|\cdot\|_{L_{2}(G, \mathcal{M})}=\left\|\langle\cdot, \cdot\rangle_{L_{2}(G, \mathcal{M})}\right\|_{\mathcal{A}}^{\frac{1}{2}}
$$


and

$$
\|\cdot\|_{L_{2}(\widehat{G}, \mathcal{M})}=\left\|\langle\cdot, \cdot\rangle_{L_{2}(\widehat{G}, \mathcal{M})}\right\|_{\mathcal{A}}^{\frac{1}{2}}
$$

on $L_{2}(G, \mathcal{M})$ and on $L_{2}(\widehat{G}, \mathcal{M})$ respectively, [8].

Proposition 3.10. Let $G$ be a discret abelian group. Then the map $L_{2}(G, \mathcal{M}) \rightarrow$ $L_{2}(\widehat{G}, \mathcal{M}), f \mapsto \widehat{f}$ is a linear isometry.

Proof. Let $f \in L_{1}(G, \mathcal{M}) \cap L_{2}(G, \mathcal{M})$. Then

$$
\begin{aligned}
\int_{\widehat{G}}\|\widehat{f}(\chi)\|_{\mathcal{M}}^{2} d \Lambda(\chi) & =\int_{\widehat{G}}\|\langle\widehat{f}(\chi), \widehat{f}(\chi)\rangle\|_{\mathcal{A}} d \Lambda(\chi) \\
& =\int_{\widehat{G}}\left\|\left\langle\int_{G} f(t) \overline{\chi(t)} d \lambda(t), \int_{G} f(s) \overline{\chi(s)} d \lambda(s)\right\rangle\right\|_{\mathcal{A}} d \Lambda(\chi) \\
& =\int_{\widehat{G}}\left\|\int_{G} \int_{G}\langle f(t), f(s)\rangle \chi(t) \overline{\chi(s)} d \lambda(t) d \lambda(s)\right\|_{\mathcal{A}} d \Lambda(\chi) \\
& \leqslant \int_{\widehat{G}}\left(\int_{G} \int_{G}\|\langle f(t), f(s)\rangle \chi(t) \overline{\chi(s)}\|_{\mathcal{A}} d \lambda(t) d \lambda(s)\right) d \Lambda(\chi) \\
& =\int_{\widehat{G}}\left(\int_{G} \int_{G}\|\langle f(t), f(s)\rangle\|_{\mathcal{A}}|\chi(t) \overline{\chi(s)}| d \lambda(t) d \lambda(s)\right) d \Lambda(\chi) \\
& =\int_{\widehat{G}}\left(\int_{G} \int_{G}\|\langle f(t), f(s)\rangle\|_{\mathcal{A}} d \lambda(t) d \lambda(s)\right) d \Lambda(\chi) \\
& =\Lambda(\widehat{G}) \int_{G} \int_{G}\|\langle f(t), f(s)\rangle\|_{\mathcal{A}} d \lambda(t) d \lambda(s) \\
& \leqslant \Lambda(\widehat{G}) \int_{G} \int_{G}\|f(t)\|_{\mathcal{M}}\|f(s)\|_{\mathcal{M}} d \lambda(t) d \lambda(s) \\
& =\Lambda(\widehat{G})\left(\int_{G}\|f(t)\|_{\mathcal{M}} d \lambda(t)\right)\left(\int_{G}\|f(s)\|_{\mathcal{M}} d \lambda(s)\right) \\
& =\Lambda(\widehat{G})\left(\int_{G}\|f(t)\|_{\mathcal{M}} d \lambda(t)\right)^{2}<+\infty .
\end{aligned}
$$

Hence $\widehat{f} \in L_{2}(\widehat{G}, \mathcal{M})$ and since $L_{1}(G, \mathcal{M}) \cap L_{2}(G, \mathcal{M})$ is dense in $L_{2}(G, \mathcal{M})$ it follows that the Fourier transform can be extended in one way to the map $L_{2}(G, \mathcal{M}) \rightarrow$ $L_{2}(\widehat{G}, \mathcal{M}), f \mapsto \widehat{f}$.

Let $f \in L_{2}(G, \mathcal{M})$. We have

$$
\|\widehat{f}\|_{L_{2}(\widehat{G}, \mathcal{M})}=\left\|\langle\widehat{f}, \widehat{f}\rangle_{L_{2}(\widehat{G}, \mathcal{M})}\right\|_{\mathcal{A}}^{\frac{1}{2}}=\left\|\langle f, f\rangle_{L_{2}(G, \mathcal{M})}\right\|_{\mathcal{A}}^{\frac{1}{2}}=\|f\|_{L_{2}(G, \mathcal{M})} .
$$

Let $g \in L_{2}(\widehat{G}, \mathcal{M})$, the inverse Fourier transform is defined for all $t \in G$ by

$$
\check{g}(t)=\int_{\widehat{G}} \chi(t) g(\chi) d \Lambda(\chi) .
$$


Proposition 3.11. Let $G$ be a discret abelian group, then for $f \in L_{2}(G, \mathcal{M}), \check{\hat{f}}=f$. Proof. Let $f \in L_{2}(G, \mathcal{M})$, for all $t \in G$, we have

$$
\begin{aligned}
\check{\hat{f}}(t) & =\int_{\widehat{G}} \chi(t) \widehat{f}(\chi) d \Lambda(\chi) \\
& =\int_{\widehat{G}} \chi(t)\left(\int_{G} f(s) \overline{\chi(s)} d \lambda(s)\right) d \Lambda(\chi) \\
& =\int_{\widehat{G}} \int_{G} \chi(t) \overline{\chi(s)} f(s) d \lambda(s) d \Lambda(\chi) \\
& =\int_{\widehat{G}} \int_{G} \delta_{t}(\chi) \overline{\delta_{s}(\chi)} f(s) d \lambda(s) d \Lambda(\chi) \\
& =\int_{G} f(s)\left(\int_{\widehat{G}} \delta_{t}(\chi) \overline{\delta_{s}(\chi)} d \Lambda(\chi)\right) d \lambda(s) \\
& =\int_{G} f(s)\left\langle\delta_{t}, \delta_{s}\right\rangle \widehat{\widehat{G}} d \lambda(s) \\
& =\int_{G} f(s) \delta_{s, t} d \lambda(s) \\
& =f(t) .
\end{aligned}
$$

Proposition 3.12. Let $G$ be a compact abelian group, then for $g \in \mathcal{C}_{0}(\widehat{G}, \mathcal{M}) \cap$ $L_{2}(\widehat{G}, \mathcal{M})$, we have $\widehat{g}=g$.

Proof. Let $\chi \in \widehat{G}$. We have

$$
\begin{aligned}
\widehat{\hat{g}}(\chi) & =\int_{G} \overline{\chi(t)} \check{g}(t) d \lambda(t) \\
& =\int_{G} \overline{\chi(t)}\left(\int_{\widehat{G}} \eta(t) g(\eta) d \Lambda(\eta)\right) d \lambda(t) \\
& =\int_{G}\left(\int_{\widehat{G}} \overline{\chi(t)} \eta(t) g(\eta) d \Lambda(\eta)\right) d \lambda(t) \\
& =\int_{\widehat{G}} g(\eta)\left(\int_{G} \overline{\chi(t)} \eta(t) d \lambda(t)\right) d \Lambda(\eta) \\
& =\int_{\widehat{G}} g(\eta)\langle\chi, \eta\rangle_{\widehat{G}} d \Lambda(\eta) \\
& =g(\chi) .
\end{aligned}
$$

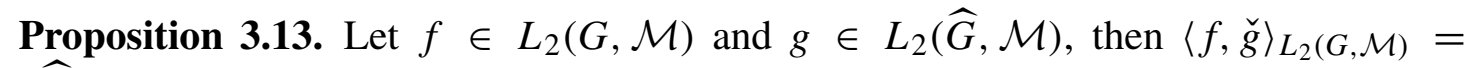
$\langle\widehat{f}, g\rangle_{L_{2}(\widehat{G}, \mathcal{M})}$. 
Proof. Let $f \in L_{2}(G, \mathcal{M})$ and $g \in L_{2}(\widehat{G}, \mathcal{M})$. We have

$$
\begin{aligned}
\langle f, \check{g}\rangle_{L_{2}(G, \mathcal{M})} & =\int_{G}\langle f(t), \check{g}(t)\rangle d \lambda(t) \\
& =\int_{G}\left\langle f(t), \int_{\widehat{G}} \chi(t) g(\chi) d \Lambda(\chi)\right\rangle d \lambda(t) \\
& =\int_{G} \int_{\widehat{G}}\langle f(t), \chi(t) g(\chi)\rangle d \Lambda(\chi) d \lambda(t) \\
& =\int_{G} \int_{\widehat{G}} \chi(t)\langle f(t), g(\chi)\rangle d \Lambda(\chi) d \lambda(t) \\
& =\int_{G} \int_{\widehat{G}}\langle\overline{\chi(t)} f(t), g(\chi)\rangle d \Lambda(\chi) d \lambda(t) \\
& =\int_{\widehat{G}}\left\langle\int_{G} \overline{\chi(t)} f(t) d \lambda(t), g(\chi)\right\rangle d \Lambda(\chi) \\
& =\int_{\widehat{G}}\langle\widehat{f}(\chi), g(\chi)\rangle d \Lambda(\chi) \\
& =\langle\widehat{f}, g\rangle_{L_{2}(\widehat{G}, \mathcal{M})} .
\end{aligned}
$$

In Hilbert $C^{*}$-module-valued functions settings we set the following definition for the convolution product.

Definition 3.14. Let $f, g \in L_{1}(G, \mathcal{M}), f \otimes g$ is defined by

$$
f \otimes g(t)=\int_{G}\left\langle f\left(x t^{-1}\right), g(x)\right\rangle_{\mathcal{M}} d \lambda(x) \text { for all } t \in G .
$$

Let $L_{1}(G, \mathcal{A})$ denote the space of Bochner-integrable $\mathcal{A}$-valued functions on $G$. It is a Banach space with respect to the norm

$$
\|f\|_{\mathcal{A}}^{1}=\int_{G}\|f(t)\|_{\mathcal{A}} d \lambda(t) \text { for all } f \in L_{1}(G, \mathcal{A})
$$

Proposition 3.15. If $f, g \in L_{1}(G, \mathcal{M})$ then $f \otimes g \in L_{1}(G, \mathcal{A})$ and $\|f \otimes g\|_{\mathcal{A}}^{1} \leqslant$ $\|f\|_{1}\|g\|_{1}$. 
Proof. Let $f, g \in L_{1}(G, \mathcal{M})$, we have

$$
\begin{aligned}
\|f \otimes g\|_{\mathcal{A}}^{1} & =\int_{G}\|f * g(t)\|_{\mathcal{A}} d \lambda(t) \\
& =\int_{G}\left\|\int_{G}\left\langle f\left(x t^{-1}\right), g(x)\right\rangle_{\mathcal{M}} d \lambda(x)\right\|_{\mathcal{A}} d \lambda(t) \\
& \leqslant \int_{G} \int_{G}\left\|\left\langle f\left(x t^{-1}\right), g(x)\right\rangle_{\mathcal{M}}\right\|_{\mathcal{A}} d \lambda(x) d \lambda(t) \\
& \leqslant \int_{G} \int_{G}\left\|f\left(x t^{-1}\right)\right\|_{\mathcal{M}}\|g(x)\|_{\mathcal{M}} d \lambda(x) d \lambda(t) \\
& =\int_{G}\|g(x)\|_{\mathcal{M}}\left(\int_{G}\left\|f\left(x t^{-1}\right)\right\|_{\mathcal{M}} d \lambda(t)\right) d \lambda(x) \\
& =\int_{G}\|g(x)\|_{\mathcal{M}}\left(\int_{G}\|f(y)\|_{\mathcal{M}} d \lambda(y)\right) d \lambda(x) \\
& =\int_{G}\|f(y)\|_{\mathcal{M}} d \lambda(y) \int_{G}\|g(x)\|_{\mathcal{M}} d \lambda(x) \\
& =\|f\|_{1}\|g\|_{1} .
\end{aligned}
$$

Hence follows the statement.

We obtain the following analogue of the convolution theorem.

Proposition 3.16. Let $f, g \in L_{1}(G, \mathcal{M})$, then

$$
\widehat{f \otimes g}(\chi)=\langle\widehat{f}(\chi), \widehat{g}(\chi)\rangle_{\mathcal{M}} \text { for all } \chi \in \widehat{G} .
$$

Proof. Let $f, g \in L_{1}(G, \mathcal{M})$ and $\chi \in \widehat{G}$, we have

$$
\begin{aligned}
\widehat{f \otimes g}(\chi) & =\int_{G} \bar{\chi}(t) f \otimes g(t) d \lambda(t) \\
& =\int_{G} \bar{\chi}(t)\left(\int_{G}\left\langle f\left(x t^{-1}\right), g(x)\right\rangle_{\mathcal{M}} d \lambda(x)\right) d \lambda(t) \\
& =\int_{G} \bar{\chi}\left(y^{-1} x\right) \int_{G}\langle f(y), g(x)\rangle_{\mathcal{M}} d \lambda(x) d \lambda(y) \\
& =\int_{G} \int_{G} \chi(y) \overline{\chi(x)}\langle f(y), g(x)\rangle_{\mathcal{M}} d \lambda(x) d \lambda(y) \\
& =\left\langle\int_{G} \overline{\chi(y)} f(y) d \lambda(y), \int_{G} \overline{\chi(x)} g(x) d \lambda(x)\right\rangle_{\mathcal{M}} \\
& =\langle\widehat{f}(\chi), \widehat{g}(\chi)\rangle_{\mathcal{M}} .
\end{aligned}
$$

Therefore we have $\widehat{f \otimes g}(\cdot)=\langle\widehat{f}(\cdot), \widehat{g}(\cdot)\rangle_{\mathcal{M}}$. 


\section{Acknowledgment}

Y. Mensah is grateful to TWAS for its material and financial support (Fellowship for Research and Advanced Training, FR number: 3240257263).

\section{References}

[1] Bakic D., Guljaš B., 2002, Hilbert $C^{*}$-modules over $C^{*}$-algebras of compact operators, Acta Sci. Math. (Szeged).

[2] Deitmar A., 2012, A first Course in Harmoncic Analysis, Springer.

[3] Deitmar A., Echterhoff S., 2014, Principles of Harmonic analysis, Springer International Publishing, 2nd Ed.

[4] Frank M., Larson D. R., 2002, Frames in Hilbert $C^{*}$-modules and $C^{*}$-algebras, J. Op. Theory 48, 273-314.

[5] Kaplansky I., 1953, Modules over operator algebras, American Journal of Mathematics, 75(4): 839-853.

[6] Kasparov G. G., 1982, Hilbert $C^{*}$-modules: Theorems of Stinespring and Voiculescu, J. Op. Theory, Theta Foundation, 4:133-150.

[7] Kundu S., Pratibha Garg, 2009, The compact-open topology: A new perspective, Elsevier, Topology Appl. 156, 686-696.

[8] Lance E. C., 1995, Hilbert C*-Modules, A toolkit for operator algebraists, London Math. Soc. Lecture Note Ser., vol. 210, Cambridge University Press.

[9] Landsman N. P., 1998, Lecture Notes on $C^{*}$-Algebras, Hilbert $C^{*}$-modules and Quantum Mechanics, arXiv:math-ph/9807030v1.

[10] Paschke W. L., 1973, Inner product modules over B*-algebras, Trans. Am. Math. Soc. 182, 443-468.

[11] Rieffel M. A., 1974, Induced representations of $C^{*}$-algebras, Advances in Math., Elsevier, 13(2): 176-257.

[12] Rudin W., 1962, Fourier analysis on groups, Interscience tracts pure and applied mathematics No. 12, New York.

[13] Stroppel M., 2005, Locally compact groups, European Mathematical Society, Textbooks in Mathematics.

[14] Xiao X.C., Zeng X.M., 2010, Some properties of g-frames in Hilbert $C^{*}$-modules, J. Math. Anal. Appl. 363, 399-408.

[15] Xiang Z.Q., Li Y. M., 2016, G-frames for operators in Hilbert $C^{*}$-modules, Turk J Math, 40: 453-469. 